$$
\text { 4. } \bar{N}+N \rightarrow \bar{D}+D
$$

The amplitudes for $\bar{N}+N \rightarrow \bar{D}+D$ can be expressed in terms of the channel amplitudes $A_{27}, A_{8 s}, A_{8 a}$, and $A_{1}$, which are the matrix elements for the representations $27 \leftrightarrow 27,8 s \leftrightarrow 8,8 a \leftrightarrow 8$, and $1 \leftrightarrow 1$, respectively. The decuplet $D$ represents the baryon-pion resonances; the quartet $N^{++}, N^{+}, N^{0}, N^{-}$, the triplet $Y_{1}^{+}, Y_{1}^{0}, Y_{1}^{-}$, the doublet $Z^{0}, Z^{-}$, and the singlet $\Omega^{-}$. In order to consider the exchanged system, the channel amplitudes $B_{35}, B_{27}, B_{10}$, and $B_{8}$ in the crossed channel $(\bar{N} N \mid \bar{D} D) \rightarrow(D \bar{N} \mid D \bar{N})$ are listed in Table IV.

The experimental results ${ }^{18,19}$ with $3.25-\mathrm{BeV} / c$ incident $\bar{p}$ indicate that $\sigma\left(\bar{Y}_{1}-Y_{1}^{-}\right) / \sigma\left(\bar{Y}_{1}+Y_{1}{ }^{+}\right)=7$ and $\sigma\left(\bar{Y}_{1}-Y_{1}^{-}+\bar{Y}_{1}+Y_{1}+\right) / \sigma\left(\bar{N}^{++} N^{++}\right)=10^{-2}$. Also, a necessary condition for the validity of the single-pionexchange model for $\bar{p}+p \rightarrow \bar{N}^{++} N^{++}$is satisfied, which suggests that the $B_{8}$ amplitude dominates. It is attrac-

${ }^{18}$ T. Ferbel, J. Sandweiss, H. D. Taft, M. Gailloud, T. E. Kalogeropoulos, T. W. Morris, and R. M. Lea, Phys. Rev. Letters 9, 351 (1962).

${ }^{19}$ C. Baltay, J. Sandweiss, H. Taft, B. B. Culwick, W. B. Fowler, et al., Phys. Rev. Letters 11, 32 (1963). tive to suggest that the ratio $\sigma\left(\bar{Y}_{1}-Y_{1}^{-}\right) / \sigma\left(\bar{Y}_{1}+Y_{1}{ }^{+}\right)=7$ is determined by the $B_{27}$ amplitude, which leads to the theoretical ratio 6.25 (Table IV), but this is not possible as the $B_{8}$ amplitude ( $\bar{K}^{0}$ exchange), rather than the $B_{27}$ amplitude, should dominate the reaction $\bar{p}+p \rightarrow \bar{Y}_{1}{ }^{+}$ $+Y_{1}{ }^{+}$. The preceding attempt shows that a simple analysis is not possible within the framework of $\mathrm{SU}_{3}$.

The following equalities among cross sections in $(\bar{p} n)$ collision are noted from Table IV :

$$
\begin{aligned}
& \sigma\left(\bar{N}^{+}+N^{+}\right)=\sigma\left(\bar{N}^{0} N^{-}\right)=\frac{3}{4} \sigma\left(\bar{N}^{+} N^{0}\right) \\
& \sigma\left(\bar{Y}_{1}^{+} Y_{1}^{0}\right)=\sigma\left(\bar{Y}_{1}^{0} Y_{1}^{-}\right) .
\end{aligned}
$$

These are consequences of $\mathrm{SU}_{2}$. A test of $\mathrm{SU}_{3}$ would require additional dynamical assumptions for which Table IV would be useful.

\section{ACKNOWLEDGMENTS}

The author wishes to thank many of his colleagues, particularly M. Derrick, L. Hyman, K. Itabashi, and F. Throw for valuable discussions.

\title{
S-Matrix Method for Calculation of Electromagnetic Corrections to Strong Interactions*
}

\author{
Roger F. Dashen† and Steven C. Frautschi \\ California Institute of Technology, Pasadena, California \\ (Received 27 April 1964)
}

\begin{abstract}
We develop an $S$-matrix method for calculating the effect of small perturbations on a partial-wave amplitude, and in particular, on the positions and residues of bound states. The method is applicable to both nonrelativistic and relativistic problems. It has, as a particular virtue, rapid convergence of the dispersion integrals. Electromagnetic corrections to strong interactions are the main application we have in mind, and modifications useful for handling the infrared divergence that occurs in this case are described in detail.
\end{abstract}

\section{INTRODUCTION}

$\mathrm{T}$ RADITIONALLY, electromagnetic corrections to strong interactions have been formulated in terms of off-mass-shell propagators and vertex functions. For example, the usual method for calculating the neutron-proton mass difference consists of finding the electromagnetic corrections to the nucleon propagator.

On the other hand, much recent progress in "strong interaction dynamics has come from a study of the two-body scattering amplitude on the mass shell. In this paper we use the on-mass-shell, $S$-matrix formalism

* Work supported in part by the U. S. Atomic Energy Commission. The work reported here is included in a thesis to be submitted by Roger F. Dashen to the California Institute of Technology in partial fulfillment of the requirements for the degree of Doctor of Philosophy.

$\uparrow$ National_Science Foundation_Predoctoral Fellow. to study electromagnetic effects, including corrections to masses and coupling constants.

We feel that this approach has several advantages: (i) According to the "bootstrap" hypothesis, all strongly interacting particles are bound states or resonances. From this point of view, the mass differences among the members of an isotopic multiplet result from electromagnetic corrections to the interactions which hold the particles together. Now, in $S$-matrix studies, closely related methods apply to both nonrelativistic and relativistic problems; one can therefore use the understanding of bound states, resonances, and perturbations on the interaction that one has in nonrelativistic quantum mechanics as a guide in relativistic problems which, according to the "bootstrap" hypothesis, possess these same features. (ii) The customary approximation scheme in strong interactions emphasizes the long-range parts of the 
interaction. These are the best understood parts, and there exists ample evidence attesting to their importance. This approximation scheme again appears suitable for estimating corrections due to electromagnetic interactions, which are of even longer range. It is straightforward to distinguish long-range effects in the $S$-matrix approach, and it seems likely that the dispersion integrals for the $S$ matrix are less sensitive to short-range or high-mass corrections than are the integrals appearing in propagators or vertex functions.

In the following paper by Dashen, these features are illustrated by an application to the neutron-proton mass difference. Neutrons and protons are considered as bound-state poles in the $\pi$ - $N$ scattering amplitude, and the mass difference is estimated by finding the difference in their binding energies. The results are in excellent agreement with experiment. As pointed out above, we expect that, in general, long-range effects will play a very important role in the $S$ matrix. One indeed finds that the dispersion integrals for the $p-n$ mass difference are almost completely dominated by the longest range correction to the $\pi-N$ interaction, namely photon exchange.

There are a number of other possible applications of our formalism which would be of immediate experimental interest. Among the most interesting would be the mass splittings in the other isospin multiplets. Such calculations, however, depend on parameters which are less well known for most multiplets than for pions and nucleons. For example, one would presumably have to known the $\Sigma$ magnetic moments in order to compute the $\Sigma$ mass differences. Further possible applications include the electromagnetic corrections to the $\pi-N$ coupling constants and the low-energy $\pi-N$ phase shifts. Here, our detailed knowledge of pions and nucleons would give the necessary parameters, but the amount of labor involved would be somewhat greater than that required to find mass differences. Similarly, one could obtain estimates for the corrections to lowenergy $N-N$ scattering. Actually, our methods are not restricted to electromagnetic problems, and might prove useful in potential theory and other contexts.

In the next section we develop the formalism within the framework of nonrelativistic potential scattering and then, in Sec. III, the method is carried over into the relativistic domain. A large part of the paper is devoted to a discussion of infrared divergences and how they can be circumvented in the most advantageous manner. The reader who is not particularly interested in the details of how the infrared terms are handled may find it advisable to read only through Eq. (6) in Sec. II and Eq. (24) in Sec. III of the present paper, and then proceed to the treatment of the neutron-proton mass difference, where our techniques are illustrated in the context of a practical physical problem.

\section{POTENTIAL SCATTERING}

Our first task is to develop, within the framework of nonrelativistic potential scattering, a perturbation theory which can be extended to relativistic problems. For simplicity, we consider only $S$-wave scattering and define the amplitude

$$
A(s)=e^{i \eta} \sin \eta / q,
$$

where $\eta$ is the phase shift, $q$ is the momentum, and $s=q^{2}$. We take the mass of the particle to be $\frac{1}{2}$ so that $s$ is the kinetic energy. ${ }^{1}$ It is known ${ }^{2}$ that, for a superposition of Yukawa potentials, $A$ is an analytic function of $s$ with a right-hand cut required by unitarity and a left-hand cut which comes from the partial-wave projections of the Born amplitude and double spectral function. Given the discontinuity across the left cut, one can use the $N / D$ method to obtain the amplitude $A$.

We will suppose that for some strong potential $V$, the amplitude has been obtained in the form $N / D$, and derive an expression for the first-order change $\delta A$ in the amplitude when the problem is perturbed by an additional weak potential $\delta V$. Let us also assume that the unperturbed problem has a bound state at $s=s_{B}$ so that $D\left(s_{B}\right)=0$ and $A \sim R / s-s_{B}$ near $s_{B}$, and ask for the first-order change in the position and residue $\delta s_{B}$ and $\delta R$ of the bound-state pole. By definition, we have

$$
\delta A=(\delta \eta / q) e^{2 i \eta}
$$

along the right cut and

$\delta A \sim \delta\left(R / s-s_{B}\right)=\left[R /\left(s-s_{B}\right)^{2}\right] \delta s_{B}+\left[\delta R /\left(s-s_{B}\right)\right]$

near the bound-state pole. Since the square of the unperturbed denominator function $D^{2}$ has the phase $e^{-2 i \eta}$ along the right-hand cut and a double zero at $s=s_{B}$, the function $D^{2} \delta A$ has no poles or right-hand cut. The denominator function can be chosen ${ }^{2}$ such that $D$ tends to a constant as $s \rightarrow \infty$, and a simple application of Cauchy's theorem yields

$$
\delta A(s)=\frac{1}{D^{2}(s)} \frac{1}{\pi} \int_{-\infty}^{0} \frac{D^{2}\left(s^{\prime}\right) \operatorname{Im} \delta A\left(s^{\prime}\right)}{s^{\prime}-s} d s^{\prime},
$$

where we have used the fact that $D$ has no left cut. Picking out the coefficients of the poles in (3), we find

$$
\begin{gathered}
\delta s_{B}=\frac{1}{R\left(D^{\prime}\left(s_{B}\right)\right)^{2}} \frac{1}{\pi} \int_{-\infty}^{0} \frac{D^{2}\left(s^{\prime}\right) \operatorname{Im} \delta A\left(s^{\prime}\right)}{s^{\prime}-s_{B}} d s^{\prime} \\
\delta R=\frac{-2 R D^{\prime \prime}\left(s_{B}\right) \delta s_{B}}{D^{\prime}\left(s_{B}\right)}+\frac{1}{\left(D^{\prime}\left(s_{B}\right)\right)^{2} \pi}-\frac{1}{2} \\
\quad \times \int_{-\infty}^{0} \frac{D^{2}\left(s^{\prime}\right) \operatorname{Im} \delta A\left(s^{\prime}\right)}{\left(s^{\prime}-s_{B}\right)^{2}} d s^{\prime} .
\end{gathered}
$$

${ }^{1}$ We take $\hbar=c=1$ in addition to setting the kinetic energy equal to $q^{2}$.

${ }^{2} \mathrm{R}$. Blankenbecler, M. Goldberger, N. Khuri, and S. Treiman, Ann. Phys. (N. Y.) 10, 62 (1960). 
Note that the reality of $\delta s_{B}$ and $\delta R$ is guaranteed by the vanishing of $D\left(s^{\prime}\right)$ at $s^{\prime}=s_{B}$.

Equations (2) to (6) are completely adequate for treating short-range perturbations. We are developing the nonrelativistic formalism in this section, however, primarily for purposes of orientation or introduction to the calculation of electromagnetic corrections to the strong interactions. Electromagnetic corrections are complicated by the infrared divergence associated with the massless nature of the photon, which requires a modification of the formalism presented thus far. To find the appropriate modification, it is convenient to study a nonrelativistic perturbation which has essential features of the relativistic problem, including the infrared divergence. Thus, we consider a perturbing potential whose Fourier transform is proportional to $\left(1 / t-\lambda^{2}\right)\left(m^{2} / t-m^{2}\right)^{2}$ where $t=-2 q^{2}(1-\cos \theta)$. Potentials of this type are characteristic of the "singlephoton exchange" potential between two strongly interacting particles whose form factors obey unsubtracted dispersion relations. The constant $\lambda$ is a fictitious photon mass which must eventually be set equal to zero.

The spatial form of the above potential is

$$
\delta V(r)=b\left[e^{-\lambda r} / r-e^{-m r} / r+\left(\left(\lambda^{2}-m^{2}\right) / 2 m\right) e^{-m r}\right],
$$

where we have introduced a strength parameter $b$ which we assume to be small. If the photon mass $\lambda$ is set equal to zero, Eq. (7) becomes a modified Coulomb potential. As is well known, in the limit $\lambda \rightarrow 0$, logarithmic divergences will appear in the right-hand side of Eq. (4) for $\delta A$. This is, of course, due to the infinite range of the Coulomb potential. From elementary quantum mechanics, one knows that, when the perturbation is summed to all orders, the divergent part contributes to the phase shift a term proportional to $\ln (q r)$, or equivalently, $\ln (q / \lambda)$.

The divergent term is common to all partial waves. Thus, above threshold it appears only as a phase factor $\exp [-(i b / q) \ln q r]$ which multiplies the entire $S$ matrix and has no observable effect. We find it expedient to abandon $\delta A$ and choose a new amplitude $\delta \hat{A}$ which does not contain this divergent unobservable phase factor for the following reasons:

(i) The infrared factor introduces an $r$ dependence into the $S$-matrix element $e^{2 i \eta}$, which would otherwise be independent of $r$. In a perturbation treatment, this $r$ dependence appears as a logarithmic divergence in $\delta A$. (ii) Below threshold $q$ becomes $i|q|$, and the $r$ dependence takes the form $\exp [-(b /|q|) \ln (|q| r)]$ $=(|q| r)^{-b /|q|}$. The residue $R$ of a bound-state pole will therefore contain a factor which is either zero or infinity, making $\delta R$ infinite in a perturbation treatment. It is convenient to remove such factors by a redefinition of the amplitude, leading to a redefined residue that depends only on finite quantities. (iii) Unlike the residue $R$, the binding energy $s_{B}$ should not have an infrared divergence. In an approximate evaluation of Eq. (5) for $\delta s_{B}$, however, a spurious divergence is likely to appear. Again, this difficulty can be avoided by a redefinition of the amplitude.

The redefinition we shall employ is obtained by removing from the $S$ matrix the infrared divergent factor $\exp \left[-i(b / 2 q) \ln \left(g(s) / \lambda^{2}\right)\right]$, where $g(s)$ is an as yet unspecified function with dimension (mass) ${ }^{2}$. One readily verifies that $\delta \hat{A}$ is then given by $\delta \hat{A}=\delta A$ $+\left(b / 4 q^{2}\right) e^{2 i \eta} \ln \left(g(s) / \lambda^{2}\right)$. For any $g(s)$, the resulting amplitude is well behaved in the limit $\lambda \rightarrow 0$. We shall make use of this freedom by choosing $g(s)$ in a way that minimizes the sensitivity of our dispersion relations to distant singularities, which are generally less well known than the nearby ones.

It turns out that the best choice for $g(s)$ corresponds to the following $\delta \hat{A}$ :

$$
\begin{aligned}
\delta \hat{A} & =\delta \hat{\eta}\left(e^{2 i \eta} / q\right)=\left(\delta \eta-\delta \eta_{\mathrm{Born}}\right)\left(e^{2 i \eta} / q\right), \\
\delta \eta_{\mathrm{Born}} & \equiv-\frac{1}{q} \int_{0}^{\infty} \sin ^{2}(q r) \delta V(r) d r .
\end{aligned}
$$

Since $\delta \eta_{\text {Born }}$ contains the same $\ln \lambda$ dependence as $\delta \eta$, the infrared phase shift is indeed removed from $\delta \hat{A}$, which is therefore well behaved in the limit $\lambda \rightarrow 0$. One will also note that $\delta \hat{A}$ has the correct threshold behavior. It remains to show that, for perturbing potentials of the form (7), $\delta \hat{A}$ should be quite insensitive to the distant singularities in the dispersion integrals.

The argument about distant singularities runs as follows: For short-range $V$ we can, to a good approximation, write

$$
\delta \eta=-\frac{1}{q} \int_{0}^{\infty} \sin ^{2}(q r+\eta) \delta V(r) d r .
$$

Performing a few algebraic manipulations, one finds

$$
\begin{aligned}
\delta \hat{\eta}=\delta \eta-\delta \eta_{\mathrm{Born}}=-\sin ^{2} \eta & \int_{0}^{\infty} \frac{\cos 2 q r}{q} \delta V(r) d r \\
& -\frac{1}{2} \sin 2 \eta \int_{0}^{\infty} \frac{\sin 2 q r}{q} \delta V(r) d r .
\end{aligned}
$$

For the perturbing potential (7) with $\lambda=0$ we find, for $|q| \gg m$,

$$
\delta \hat{\eta}=-\left(b m \sin 2 \eta / 8 q^{2}\right)+O\left(b m^{2} / q^{3}\right),
$$

which is a more rapid falloff at large $q$ than that of $\delta \eta_{\text {Born }}(\sim 1 / q)$. Thus, as successively more distant singularities are included in a dispersion-theoretic calculation, $\delta \hat{A}$ is expected to converge more rapidly than would the subtracted term $\left(\delta \eta_{\mathrm{Born}} / q\right) e^{2 i \eta}$.

Equation (12) was obtained from a rather special model, and one naturally wonders which features of the result are general. It is possible, using various simple 
forms for $V$ and $\delta V$, to show that the good convergence of $\delta \hat{A}$ can hold even when $V$ is not restricted to short range. The rapid convergence does depend critically, however, on the "smooth" $r$ dependence of $\delta V$ which, as given by Eq. (7), is finite at the origin. If more singular behavior at the origin is admitted, or $\delta V$ is given a discontinuous behavior at some other $r, \delta \hat{\eta}$ falls off more slowly at large $q$. This is in accord with the physical notion that a rapid spatial variation corresponds to high Fourier components in $q$. Of course, we do not know the small-distance behavior of electromagnetic corrections to the strong interaction, but at least the apparently rapid convergence of the neutronproton mass difference calculation in the following paper is consistent with a smooth cutoff $\delta V$ at intermediate range. The one contribution to the really short-range potential on which detailed information is available is one-photon exchange, and here the recent Cambridge Electron Accelerator data ${ }^{3}$ on the proton form factor indicates a smooth behavior down to very small distances.

Another possible reason for rapid convergence of $\delta \hat{A}$ is that in potential theory any phase shift tends to its Born approximation at high energy. Thus, when potential theory is applicable, $\left(\delta \eta-\delta \eta_{\text {Born }}\right)$ approaches zero faster than $\delta \eta_{\text {Born }}$ at large $s$, independently of whether $\delta V$ is cut off at small distances. This argument is less satisfactory than the arguments of the previous paragraph, however, because (i) it does not imply that $\delta \eta$ approaches $\delta \eta_{\text {Born }}$ any faster than the unperturbed phase shift $\eta$ approaches its Born value; and (ii) unlike the potentials in ordinary potential theory, the strong interaction depends on energy, with the result that $\eta$ may approach the Born approximation very slowly if at all. We therefore prefer to rely primarily on the fact that when $\delta V$ is cut off at small distances, as in Eq. (12), $\delta \hat{\eta}$ falls off rapidly at large $q$ no matter what asymptotic behavior the strong interaction phase shift $\eta$ may have.

The procedure we have adopted of dropping the part of $\delta A$ which diverges like $\ln \left(\lambda^{2} / g(s)\right)$ is roughly equivalent to giving the photon a mass $g$. Our method differs from handling the infrared divergence by a photon mass, however, because $g(s)$ depends on energy. The energy dependence is needed to make the dispersion relation for the redefined amplitude converge rapidly.

It is simple enough to rephrase the dispersion relations in terms of $\delta \hat{A}$. Using the fact that $D^{2} \delta \hat{A}$, like $D^{2} \delta A$, has no poles or right-hand cut, one readily verifies that Eq. (4) continues to hold if $\delta A$ is everywhere replaced by $\delta \hat{A}$. Furthermore, since $\delta \hat{A}$ has the same double pole $R \delta s_{B} /\left(s-s_{B}\right)^{2}$ as $\delta A$ [cf. Eq. (3)], one can replace $\operatorname{Im} \delta A\left(s^{\prime}\right)$ by $\operatorname{Im} \delta \hat{A}\left(s^{\prime}\right)$ in the right-hand side of Eq. (5) for $\delta s_{B}$. Finally, if we define $\delta \hat{R}$ as the

${ }^{3}$ K. W. Chen, A. A. Cone, J. R. Dunning, Jr., S. G. F. Frank, N. F. Ransey, J. K. Walker, and Richard Wilson, Phys. Rev. Letters 11, 561 (1963). coefficient of the simple pole in $\delta \hat{A}$ [cf. Eq. (3)], then $\delta \hat{R}$ is given by (6) with $\operatorname{Im} \delta A\left(s^{\prime}\right)$ replaced by $\operatorname{Im} \delta \hat{A}\left(s^{\prime}\right) .^{4}$

The following simple example will illustrate a number of the points discussed above. The example involves a comparison between a standard method for calculating $\delta s_{B}$ and the $S$-matrix method of the present paper. The standard expression for $\delta s_{B}$ is given by

$$
\delta s_{B}=\int_{0}^{\infty}|\Psi|^{2} \delta V d r
$$

where $\psi$ is the unperturbed wave function. We take (7) for $\delta V$ and, for simplicity, assume that $V$ is of very short range. For short-range $V$, we can set

$$
|\psi|^{2} \approx 2\left|s_{B}\right|^{1 / 2} \exp \left(-2\left|s_{B}\right|^{1 / 2} r\right)
$$

and performing the integration in (13) with $\lambda=0$, we find

$$
\delta s_{B} \approx 2 b\left|s_{B}\right|^{1 / 2}\left(\ln \left(\frac{m+2\left|q_{B}\right|}{2\left|q_{B}\right|}\right)-\frac{m}{2 m+4\left|q_{B}\right|}\right) .
$$

Next we make an independent calculation of $\delta s_{B}$ using the dispersion relation (5) with $\operatorname{Im} \delta A\left(s^{\prime}\right)$ replaced by $\operatorname{Im} \delta \hat{A}\left(s^{\prime}\right)$. We will keep only the nearby singularities in the dispersion integral, and then compare the results with (15). Since $V$ is of short range, the only nearby singularities in $\delta \hat{A}=\left[\delta A-\left(\delta \eta_{\mathrm{Born}} / q\right) e^{2 i \eta}\right]$ will come from the Born term in $\delta A$ and from the singularities in $\delta \eta_{\mathrm{Born}}$. Using $\delta A_{\mathrm{Born}} \equiv \delta \eta_{\mathrm{Born}} / q$, we find

$$
\begin{aligned}
\operatorname{Im} \delta \hat{A}=\left(1-e^{2 i \eta}\right) \operatorname{Im}\left(\delta \eta_{\mathrm{Born}} / q\right)=- & 2 i q\left(e^{i \eta} \sin \eta / q\right) \\
& \times \operatorname{Im}\left(\delta \eta_{\mathrm{Born}} / q\right)
\end{aligned}
$$

along the nearby part of the left cut. For the perturbing potential (7), it turns out that all of the singularities in $\delta \eta_{\text {Born }} / q$ lie in the interval $-\frac{1}{4} m^{2} \leq s \leq 0$. Using Eq. (5) and the relations $e^{i \eta} \sin \eta / q=N / D$ and $q=i|q|$, we can then write

$$
\begin{aligned}
\delta s_{B} \approx \frac{1}{R\left(D^{\prime}\left(s_{B}\right)\right)^{2}} \frac{2}{\pi} \int_{-m 2 / 4}^{0} \mid & \left|q^{\prime}\right| \frac{N\left(s^{\prime}\right) D\left(s^{\prime}\right)}{s^{\prime}-s_{B}} \\
& \times \operatorname{Im}\left(\delta \eta_{\text {Born }}\left(s^{\prime}\right) / q^{\prime}\right) d s^{\prime} .
\end{aligned}
$$

From the effective range approximation

$$
e^{i \eta} \sin \eta / q \approx 1 /\left(i q_{B}-i q\right),
$$

${ }^{4}$ The favorable convergence of dispersion relations involving the redefined amplitude has nothing particular to do with the fact that we started with an infrared-divergent amplitude. This suggests the following treatment for other perturbation problems which have no infrared divergence: (i) Calculate $\delta \eta_{\text {Born }}$. (ii) Define $\delta \hat{A}$ as in Eq. (8). Calculate the dispersion relations for $\delta \hat{A}, \delta s_{B}$, and $\delta \hat{R}$; which converge more rapidly than the dispersion relations involving the usual amplitude. (iii) The mass shift is correctly obtained in this way. Naturally, we do not want a redefined amplitude or residue as the final answer, but to obtain the final expressions for $\delta A$ and $\delta R$, one simply adds the known $\delta \eta_{\text {Born }}$ term back in. 


\section{B 1194}

R. F. DASHEN A N D

which, for short-range $V$, should be good over the range of integration in (17); we extract $N=1, D=i q_{B}-i q$, $R=-2\left|q_{B}\right|$, and $D^{\prime}\left(s_{B}\right)=-\frac{1}{4}\left|q_{B}\right|$. The imaginary part of $\delta \eta_{\text {Born }} / q$ is known explicitly, and it turns out that the integral in (17) can be done analytically. The result is that our $S$-matrix estimate (17) for $\delta s_{B}$ is exactly the same as that given by (15). Notice two important points here. (i) We have obtained this result by keeping only the nearest singularity in $\delta \hat{A}$. (ii) As can be easily verified, if we had tried to estimate $\delta s_{B}$ by using $\operatorname{Im} \delta A\left(s^{\prime}\right)$ instead of $\operatorname{Im} \delta \hat{A}\left(s^{\prime}\right)$ in (5), a spurious infrared divergence would have appeared in our expression for $\delta s_{B}$. In a more complete calculation, such a divergence would, of course, be cancelled by a divergent term coming from more distant singularities.

\section{ELECTROMAGNETIC CORRECTIONS TO THE STRONG INTERACTIONS}

The partial-wave scattering amplitudes which appear in relativistic $S$-matrix theory are believed to obey dispersion relations similar to those which occur in potential scattering theory and, with a few modifications, the results of the previous section will be applicable to relativistic problems. For simplicity, we consider only elastic scattering of two spinless particles of equal mass. The partial-wave scattering amplitude then becomes

$$
A(s)=\rho(s) e^{i \eta} \sin \eta,
$$

where $s$ is the total center-of-mass energy squared and $\rho$ is a function which removes the kinematic singularities. [ $\rho$ is the analog of the factor $1 / q$ in (1). Note that $s$ has been given a new definition.] The phase shift $\eta$ is in general complex and a measure of the inelasticity is given by

$$
I(s)=\frac{1}{2} \rho\left|e^{2 i \eta}\right|
$$

We assume that $A$ is an analytic function of $s$ with a right-hand cut controlled by unitarity in the $s$ channel and a left-hand cut controlled by unitarity in the $t$ and $u$ channels. In order to apply the $N / D$ method, we must now be given both $\operatorname{Im} A$ along the left cut, and $I$, or equivalently, $\operatorname{Im} \eta$ along the right cut as input information.

Again we assume that the strong-interaction amplitude $A$ has been obtained in the form $N / D$, and ask for the first-order change in $A$ when the electromagnetic corrections are added to the input. We also assume that the unperturbed and perturbed problems have bound-state poles at $s_{B}$ and $s_{B}+\delta s_{B}$ with residues $R$ and $R+\delta R$, respectively.

In potential theory, the function $D^{2} \delta A$ had no righthand cut, but it acquires one in strong-interaction problems because $\delta \eta$ has an imaginary part, and also because the electromagnetic mass shifts of the scattered particles change the kinematic factor by $\delta \rho$. The analog of Eqs. (4) to (6) becomes

$$
\begin{aligned}
& \delta A(s)=\frac{1}{D^{2}(s)}-\left[\int_{L} \frac{D^{2}\left(s^{\prime}\right) \operatorname{Im} \delta A\left(s^{\prime}\right)}{s^{\prime}-s} d s^{\prime}\right. \\
& \left.+\int_{R} \frac{\operatorname{Im}\left[D^{2}\left(s^{\prime}\right) \delta A\left(s^{\prime}\right)\right]}{s^{\prime}-s} d s^{\prime}\right], \\
& \delta s_{B}=\frac{1}{R\left[D^{\prime}\left(s_{B}\right)\right]^{2}}-1 \int_{L}^{1} \frac{D^{2}\left(s^{\prime}\right) \operatorname{Im} \delta A\left(s^{\prime}\right)}{s^{\prime}-s_{B}} d s^{\prime} \\
& \left.+\int_{R} \frac{\operatorname{Im}\left[D^{2}\left(s^{\prime}\right) \delta A\left(s^{\prime}\right)\right]}{s^{\prime}-s_{B}} d s^{\prime}\right], \\
& \delta R=\frac{-2 D^{\prime \prime}\left(s_{B}\right) R \delta s_{B}}{D^{\prime}\left(s_{B}\right)}+\frac{1}{\left[D^{\prime}\left(s_{B}\right)\right]^{2}} \frac{1}{\pi} \\
& \times\left[\int_{L} \frac{D^{2}\left(s^{\prime}\right) \operatorname{Im} \delta A\left(s^{\prime}\right)}{\left(s^{\prime}-s_{B}\right)^{2}} d s^{\prime}\right. \\
& \left.+\int_{R} \frac{\operatorname{Im}\left[D^{2}\left(s^{\prime}\right) \delta A\left(s^{\prime}\right)\right]}{\left(s^{\prime}-s_{B}\right)^{2}} d s^{\prime}\right],
\end{aligned}
$$

where the integrals $R$ and $L$ run over the left and right cuts and we have assumed that $D^{2} \delta A \rightarrow 0$ for large $s$. Along the right-hand cut,

$$
\begin{aligned}
\operatorname{Im}\left(D^{2} \delta A\right) & =\operatorname{Im}\left[D^{2}\left(\delta I e^{2 i \operatorname{Re} \eta} / i-\delta \rho / 2 i\right)\right] \\
& =-|D|^{2} \delta I+\frac{1}{2} \operatorname{Re}\left(D^{2} \delta \rho\right)
\end{aligned}
$$

Calculation of the new kinematic and inelasticity corrections will be straightforward, with no difficulty of principle.

Whenever the scattered particles are charged, infrared divergences occur and cause us to redefine the amplitude. The redefinition will be carried out in the same spirit as in potential scattering, and we shall mention only the new features.

To begin with, in addition to the "Coulomb divergence" already encountered in potential scattering, new divergences associated with bremsstrahlung appear. The reaction of bremsstrahlung back onto two-body scattering occurs in the form of diagrams where a photon is emitted by one of the initial particles and absorbed by a particle in the final state. The net effect of all such diagrams $s^{5,6}$ is to make the amplitude for scattering without photon emission zero-expressing the fact that charge cannot be accelerated without radiating. When all the soft photon emissions are added in, however, the infrared divergence cancels and the total cross section exhibits only a mild dependence on photon emission. These reasons again call for a redefinition

${ }^{5}$ D. Yennie, S. Frautschi, and H. Suura, Ann. Phys. (N. Y.) 13, 379 (1961).

${ }^{6}$ J. Jauch and F. Rohrlich, The Theory of Photons and Electrons (Addison-Wesley Publishing Company, Inc., Cambridge, 1955). 
which will allow us to express the amplitude in terms of finite quantities, and to obtain residues, for example, which are only slightly shifted from their stronginteraction values. Since "bremsstrahlung diagrams" where a photon is emitted by initial charge line in the $s$ channel and absorbed by a final charge line, can also be interpreted in terms of final-state Coulomb interaction in the $t$ or $u$ channel, and the Coulomb interaction already required us to redefine the amplitude in potential scattering, it is hardly surprising that the "bremsstrahlung diagrams" require a redefinition of the amplitude.

A minimum requirement for the redefinition of the amplitude is removal of the $\ln \lambda$ dependence from $\delta A$. As in potential scattering, the satisfaction of this requirement leaves arbitrary the coefficient of $\lambda$ in the logarithm. We could choose the coefficient in the same manner as in potential scattering.

$$
\delta \hat{A}=\delta A-\rho \delta \eta_{\mathrm{Born}} e^{2 i \eta}
$$

Here, the Born phase shift is calculated from the electromagnetic correction to the generalized potential defined by Chew and Frautschi. ${ }^{7}$ This choice would be expected to give the best convergence of the dispersion relation. On the other hand, there are many terms to keep track of in the relativistic case, and it is tedious to have to compute the contribution of each of them to $\delta \eta_{\text {Born }}$ and make the subtraction in (25). The practical compromise which has been adopted in the following paper is to define the amplitude

$$
\alpha A^{\prime}=\delta A-\rho \delta \eta_{\text {infra }} e^{2 i \eta},
$$

where $\delta \eta_{\text {infra }}$ contains the infrared terms and the other important high-energy contributions from the diagrams in which photons connect external charge lines. The definition adopted for $\delta A^{\prime}$ is not unique, and $\delta A^{\prime}$ is not expected to converge as rapidly as $\delta \hat{A}$, but the diagrams in which photons connect external lines do provide the longest range forces and the dominant high-energy corrections, ${ }^{5}$ so relatively little is lost by using $\delta A^{\prime}$ in place of $\delta \hat{A}$.

Having redefined the amplitude, one can rephrase the dispersion relations for the residue and mass shift in terms of the new amplitude, or follow a somewhat simplified procedure which will be described in the next section.

\section{A SIMPLE METHOD FOR SUBTRACTING THE INFRARED TERM}

The mass shift and the redefined residue shift can be expressed via dispersion relations in which a term such as $\rho \delta \eta_{\text {infr }} e^{2 i \eta}$ has been subtracted out of $\delta A$ in the integrand. The subtraction procedure in the integrand can become quite tedious, however, particularly in the

\footnotetext{
${ }^{7}$ G. F. Chew and S. C. Frautschi, Phys. Rev. 124, 264 (1961).
}

relativistic case. We would like to describe a somewhat simpler way to subtract, which would give the same results in an exact calculation and can be shown to give nearly the same result in approximate calculations, such as the example at the end of Sec. II. To see how this goes, let us suppose that the input for (21) has been calculated with a small but finite photon mass $\lambda$. The function $\rho \delta \eta_{\text {in fra }}$ has the form

$$
\rho \delta \eta_{\mathrm{infra}}=f(s) \ln \left(\lambda^{2} / g(s)\right)+O(\lambda) .
$$

In the limit $\lambda \rightarrow 0$, the right-hand side of (21) must therefore be equal to $\delta A^{\prime}+e^{2 i n f}(s) \ln \left(\lambda^{2} / g(s)\right)+O(\lambda)$. Since any function which is logarithmically divergent as $\lambda \rightarrow 0$ can be uniquely separated into a part which diverges like $\ln \left(\lambda^{2} / g(s)\right)$ and a part which remains finite as $\lambda \rightarrow 0$, we can calculate $\delta \bar{A}$ by carrying out the integrations, taking the limit $\lambda \rightarrow 0$, and then dropping all terms which diverge like $\ln \left(\lambda^{2} / g(s)\right)$.

If we define $\delta R^{\prime}$ to be the residue of the simple pole in $\delta A^{\prime}$ [cf. Eq. (3)], $\delta R^{\prime}$ can be extracted from Eq. (23) by the same prescription that has been given to find $\delta A^{\prime}$ from Eq. (21).

The calculation of $\delta s_{B}$ is on a rather different footing. As we pointed out earlier, $\delta s_{B}$ should not diverge in the limit $\lambda \rightarrow 0$. That the formalism is consistent with this property can be seen by observing that, since $\delta \eta_{\text {infra }}$ has no pole at $s=s_{B}$, we have

$$
\lim _{s \rightarrow s_{B}}\left(s-s_{B}\right)^{2} \delta A^{\prime}=\lim _{s \rightarrow s_{B}}\left(s-s_{B}\right)^{2} \delta A=R \delta s_{B} .
$$

The limit involving $\delta A^{\prime}$ is not infrared divergent so $\delta s_{B}$ should be finite. The integral in Eq. (22) for $\delta s_{B}$, however, is just the infrared divergent integral of Eq. (21) for $\delta A$, evaluated at $s=s_{B}$. At the particular point $s=s_{B}$, the coefficient of the infrared divergent part of this integral must vanish, but the vanishing occurs through cancellations among long- and short-range contributions which are not enforced in an approximate evaluation of Eq. (22). The simplest way to remedy this deficiency of an approximate calculation is to drop the term containing $\ln \left(\lambda^{2} /\left|g\left(s_{B}\right)\right|\right)$, since its coefficient should have vanished anyway. ${ }^{8}$

In previous sections we have emphasized the rapid convergence of dispersion relations involving $\delta A^{\prime}$. The simple subtraction procedure of the present section is essentially equivalent and should also converge rapidly. The short-range contributions to (22), then, have little effect on $\delta s_{B}$ and act mainly to cancel the spurious infrared divergence produced by integrating only over long-range parts. By subtracting the spurious divergence in the above manner, we can avoid the hard work of calculating short-range contributions with little loss of accuracy. ${ }^{8}$ We take the absolute value of $g$ in the logarithm because Eq.
(22) does guarantee that $\delta s_{B}$ is real, so only the real part of $\ln \left(\lambda^{2} / g\left(s_{B}\right)\right)$ can appear in the spurious term. 